\title{
Assessing dental students' professional satisfaction with operative dentistry teaching and curriculum: A study in Saudi Arabia
}

Mohammed Alqarni ( $\sim$ maalqarny@kku.edu.sa )

King Khalid University College of Dentistry

\section{Research article}

Keywords: Curriculum, Dental students, Operative Dentistry, Professional satisfaction

Posted Date: July 10th, 2020

DOI: https://doi.org/10.21203/rs.3.rs-37016/v1

License: (c) (1) This work is licensed under a Creative Commons Attribution 4.0 International License. Read Full License

Version of Record: A version of this preprint was published at Medicine on June 25th, 2021. See the published version at https://doi.org/10.1097/MD.0000000000026459. 


\section{Abstract}

Background Evaluating students' professional satisfaction of operative dentistry teaching and curriculum can help in identifying their educational needs and improving the quality of the education imparted. This study aimed to assess the professional satisfaction derived by undergraduate dental students in Saudi Arabia from the operative dentistry course teaching and its curriculum.

Methods A total of 193 (109 male 56.48\%; and 84 female $43.52 \%$ ) students participated in a survey. The respondents were at the $10^{\text {th }}, 11^{\text {th }}$, and $12^{\text {th }}$ levels of the operational dentistry course in a ratio of $34.2 \%$, $32.1 \%$, and $33.7 \%$, respectively. Data were collected from survey items (18 questions) covering six areas: Learning Objectives, Course Materials, Content Relevance, Instructor knowledge, Instructor delivery and Style, and Facility and environment. Descriptive and analytical tests were performed using SPSS Software 19 with the significance level set at 0.05 .

Results A high level of satisfaction was seen among Level 10 (68.18\%), Level 11 (79.03\%), and Level 12 (86.15\%) students. Significant statistical difference was observed among Level 10 students with a lowlevel of satisfaction and a high level of satisfaction $(p<0.05)$. The percentage of satisfaction increased with the level. A high level of satisfaction was seen among both male $(78.90 \%)$ and female $(76.19 \%)$ students, with a total satisfaction level of $77.72 \%$.

Conclusion Continuous evaluation and assessment of teaching as well as curriculum can be a tool to improve the quality of education imparted, especially in clinical courses such as operative dentistry.

\section{Background}

Any training program needs evaluation for quality assurance and further improvement. ${ }^{1}$ Kirkpatrick's model of evaluation proposes four increasing levels to assess the impact of training programs. Level one (reaction) measures how the person feels about a course; level two (learning) measures the extent to which principles, facts, and techniques have been understood and absorbed; level three (behavior) measures the application of the principles and techniques acquired on the job; and level four (results) measures the ends, goals, and results desired. ${ }^{1,2}$ Monitoring students' reaction to their learning experiences is an activity increasingly being undertaken by higher education institutions. ${ }^{3}$ This initial level of evaluation should be an inherent feature of every training program because it indicates ways in which a training program can be enhanced and further developed besides building the base for higher levels of evaluation since reactions serve as a pointer to whether learning is possible. ${ }^{1,2}$ Students' satisfaction with and attitudes toward training programs are the most common indicators used to assess reaction. ${ }^{1,3}$ However, there is additional value in exploring graduates' reaction to training programs because they are less emotionally attached to the institution and are back at their workplaces where they can judge whether the knowledge and skills acquired during the program match their job requirements and responsibilities. 
Satisfaction and attitude are two indicators which help determine education quality. Satisfaction refers to the level to which students' experiences meet their expectations. ${ }^{4}$ However, attitudes refer to a mixture of beliefs, thoughts, and feelings that predispose graduates to respond in a positive or negative way toward institutions. ${ }^{5,6}$ In addition to their role in ensuring learning and teaching quality standards, the two indicators serve as a guide for students to aid their decision-making at the program/institution level and to compute institutional performance indicators. ${ }^{7}$

The format of dental education varies across the world; while some institutions have a yearly system, others follow a semester system. Several factors affect the learning process in any course, such as the nature of the student him/herself, the quality of demonstrators and teachers, exposure to course materials, laboratory, and clinical facilities, and so on. A continuous evaluation of these factors is necessary to ascertain whether the conditions are conducive to learning in the program assessed.

One of the methods for evaluating an educational system is surveying student opinions because students experience the full effect of the teaching during the course. ${ }^{8}$ As the main recipients of the educational system, evaluating student satisfaction is one of the significant components of assessing the quality of education. ${ }^{9}$

Operative dentistry is an important branch of dentistry constituting a major part of the teaching process in dental colleges. Students are introduced to operative dentistry from level 4 and this continues till level 12. There has been continuous development in the field of operative dentistry both in terms of materials and equipment as well as techniques. As with all the recent developments, continuous assessment of teaching strategy is necessary for a comprehensive evaluation of the teaching program. This study aimed to evaluate the professional satisfaction levels of undergraduate students training in operative dentistry regarding the teaching and the curriculum in the last three semesters of their dental course.

\section{Methods}

This descriptive-analytic study was conducted on level 10, level 11, and level 12 (clinical level) students from the faculty of dentistry in the academic year 2019-20. Ethical clearance for this study was obtained from the Institutional Review Board of King Khalid University College of Dentistry. The questionnaire distributed among the students consisted of six domains: Learning objectives, Course materials, Content relevance, Instructor knowledge, Instructor delivery and style, and Facility and environment. A total of 193 students participated in the survey of which 109 (56.48\%) were male and $84(43.52 \%)$ were female students. The respondents were students in levels 10,11 , and 12 of the courses and were in the ratio of $34.2 \%, 32.1 \%$, and $33.7 \%$, respectively. The participation in the study was voluntary and the students were briefed regarding the study and the questionnaire. The original questionnaire was developed by RAND staff, based on J Kirkpatrick, to evaluate the Adult Learning Principles and Training Evaluation. ${ }^{10}$ The questionnaire used in this study was slightly modified from the original to make it compatible with respect to operative dentistry teaching in dental education. The responses were to be graded 3, 2, or 1 corresponding to Agree, Somewhat Agree, or Disagree, respectively. After the questionnaires were 
collected, the data were entered into SPSS 19.0 (Inc., Chicago, III., USA); repeated measures test was used to evaluate the satisfaction level with education offered at different levels in operative dentistry teaching and curriculum.

\section{Results}

The results for all the six domains showed no statistical difference in responses in terms of gender ( $>0.05$ ) (see Table 1).

Table 1. Comparison of item responses of male and female students on the scale 


\begin{tabular}{|c|c|c|c|c|c|c|c|c|c|}
\hline Variables & Items & Male & $\%$ & Female & $\%$ & Total & $\%$ & $\mathrm{c}^{2}$ & p-value \\
\hline \multirow[t]{12}{*}{ Learning objectives } & \multicolumn{9}{|c|}{ Q1 I have understood the learning objectives of the operative course. } \\
\hline & Disagree & 0 & 0.00 & 0 & 0.00 & 0 & 0.00 & 0.0620 & 0.8030 \\
\hline & Somewhat agree & 21 & 19.27 & 15 & 17.86 & 36 & 18.65 & & \\
\hline & Agree & 88 & 80.73 & 69 & 82.14 & 157 & 81.35 & & \\
\hline & \multicolumn{9}{|c|}{ Q2 I have gained knowledge and skills consistent with the learning objectives. } \\
\hline & Disagree & 7 & 6.42 & 4 & 4.76 & 11 & 5.70 & 0.2440 & 0.8850 \\
\hline & Somewhat agree & 18 & 16.51 & 14 & 16.67 & 32 & 16.58 & & \\
\hline & Agree & 84 & 77.06 & 66 & 78.57 & 150 & 77.72 & & \\
\hline & \multicolumn{9}{|c|}{ Q3 This course has clarified my role as a student. } \\
\hline & Disagree & 0 & 0.00 & 0 & 0.00 & 0 & 0.00 & 0.0390 & 0.8430 \\
\hline & Somewhat agree & 7 & 6.42 & 6 & 7.14 & 13 & 6.74 & & \\
\hline & Agree & 102 & 93.58 & 78 & 92.86 & 180 & 93.26 & & \\
\hline \multirow[t]{12}{*}{ Course materials } & \multicolumn{9}{|c|}{ Q1 The course materials (slides, lectures, assignments, quiz, etc.) are easy to follow. } \\
\hline & Disagree & 5 & 4.59 & 3 & 3.57 & 8 & 4.15 & 0.1240 & 0.9400 \\
\hline & Somewhat agree & 14 & 12.84 & 11 & 13.10 & 25 & 12.95 & & \\
\hline & Agree & 90 & 82.57 & 70 & 83.33 & 160 & 82.90 & & \\
\hline & \multicolumn{9}{|c|}{ Q2 The complexity and level of detail of the materials are appropriate. } \\
\hline & Disagree & 2 & 1.83 & 0 & 0.00 & 2 & 1.04 & 1.5580 & 0.4590 \\
\hline & Somewhat agree & 5 & 4.59 & 4 & 4.76 & 9 & 4.66 & & \\
\hline & Agree & 102 & 93.58 & 80 & 95.24 & 182 & 94.30 & & \\
\hline & \multicolumn{9}{|c|}{$\begin{array}{l}\text { Q3 The course materials, including resources, are essential to my success in } \\
\text { operative dentistry }\end{array}$} \\
\hline & Disagree & 6 & 5.50 & 3 & 3.57 & 9 & 4.66 & 0.5750 & 0.7500 \\
\hline & Disagree & 8 & 7.34 & 5 & 5.95 & 13 & 6.74 & & \\
\hline & Somewhat agree & 95 & 87.16 & 76 & 90.48 & 171 & 88.60 & & \\
\hline \multirow[t]{12}{*}{ Content relevance } & \multicolumn{9}{|c|}{ Q1 I shall be able to apply what I learned during this course in future as a dentist. } \\
\hline & Disagree & 0 & 0.00 & 0 & 0.00 & 0 & 0.00 & 1.5570 & 0.2120 \\
\hline & Somewhat agree & 2 & 1.83 & 0 & 0.00 & 2 & 1.04 & & \\
\hline & Agree & 107 & 98.17 & 84 & 100.0 & 191 & 98.96 & & \\
\hline & \multicolumn{9}{|c|}{$\begin{array}{l}\text { Q2 I have obtained the necessary knowledge and skills to become a successful } \\
\text { dentist. }\end{array}$} \\
\hline & Disagree & 2 & 1.83 & 0 & 0.00 & 2 & 1.04 & 1.5580 & 0.4590 \\
\hline & Somewhat agree & 5 & 4.59 & 4 & 4.76 & 9 & 4.66 & & \\
\hline & Agree & 102 & 93.58 & 80 & 95.24 & 182 & 94.30 & & \\
\hline & \multicolumn{9}{|c|}{ Q3 I know where to find answers to questions that may arise in my role as a dentist. } \\
\hline & Disagree & 7 & 6.42 & 3 & 3.57 & 10 & 5.18 & 0.9760 & 0.6140 \\
\hline & Somewhat agree & 8 & 7.34 & 5 & 5.95 & 13 & 6.74 & & \\
\hline & Agree & 94 & 86.24 & 76 & 90.48 & 170 & 88.08 & & \\
\hline
\end{tabular}


Q1 My learning was enriched by the instructor's knowledge.

\begin{tabular}{|l|c|c|c|c|c|c|c|c|}
\hline Disagree & 4 & 3.67 & 2 & 2.38 & 6 & 3.11 & 0.2650 & 0.8760 \\
\hline Somewhat agree & 4 & 3.67 & 3 & 3.57 & 7 & 3.63 & & \\
\hline Agree & 101 & 92.66 & 79 & 94.05 & 180 & 93.26 & & \\
\hline
\end{tabular}

Q2 My learning was enriched by the experience of the instructor and the examples shared in the class.

Instructor delivery and

\begin{tabular}{|l|c|c|c|c|c|c|c|c|}
\hline Disagree & 5 & 4.59 & 2 & 2.38 & 7 & 3.63 & 1.2280 & 0.5410 \\
\hline Somewhat agree & 7 & 6.42 & 8 & 9.52 & 15 & 7.77 & & \\
\hline Agree & 97 & 88.99 & 74 & 88.10 & 171 & 88.60 & & \\
\hline
\end{tabular}
style

Q1 I was well engaged during the operative course.

\begin{tabular}{|l|c|c|c|c|c|c|c|c|}
\hline Disagree & 5 & 4.59 & 3 & 3.57 & 8 & 4.15 & 0.3250 & 0.8500 \\
\hline Somewhat agree & 6 & 5.50 & 6 & 7.14 & 12 & 6.22 & & \\
\hline Agree & 98 & 89.91 & 75 & 89.29 & 173 & 89.64 & & \\
\hline
\end{tabular}

Q2 I found it easy to be actively involved during the learning process.

\begin{tabular}{|l|c|c|c|c|c|c|c|c|}
\hline Disagree & 5 & 4.59 & 3 & 3.57 & 8 & 4.15 & 0.1230 & 0.9400 \\
\hline Somewhat agree & 9 & 8.26 & 7 & 8.33 & 16 & 8.29 & & \\
\hline Agree & 95 & 87.16 & 74 & 88.10 & 169 & 87.56 & & \\
\hline
\end{tabular}

Q3 I had ample opportunity to ask questions and receive answers.

\begin{tabular}{|l|c|c|c|c|c|c|c|c|}
\hline Disagree & 2 & 1.83 & 2 & 2.38 & 4 & 2.07 & 0.0740 & 0.9640 \\
\hline Somewhat agree & 5 & 4.59 & 4 & 4.76 & 9 & 4.66 & & \\
\hline Agree & 102 & 93.58 & 78 & 92.86 & 180 & 93.26 & & \\
\hline
\end{tabular}

Q4 I had ample opportunity to practice and demonstrate the skills that I leant.

\begin{tabular}{|l|c|c|c|c|c|c|c|c|}
\hline Disagree & 6 & 5.50 & 9 & 10.71 & 15 & 7.77 & 1.7970 & 0.4070 \\
\hline Somewhat agree & 7 & 6.42 & 5 & 5.95 & 12 & 6.22 & & \\
\hline Agree & 96 & 88.07 & 70 & 83.33 & 166 & 86.01 & & \\
\hline
\end{tabular}

Q5 I was comfortable with the pace of the operative sessions in the course.

\begin{tabular}{|l|c|c|c|c|c|c|c|c|}
\hline Disagree & 7 & 6.42 & 9 & 10.71 & 16 & 8.29 & 1.1630 & 0.5590 \\
\hline Somewhat agree & 9 & 8.26 & 7 & 8.33 & 16 & 8.29 & & \\
\hline Agree & 93 & 85.32 & 68 & 80.95 & 161 & 83.42 & & \\
\hline
\end{tabular}

Q6 I was comfortable with the length of the operative sessions in the course.

\begin{tabular}{|l|c|c|c|c|c|c|c|c|}
\hline Disagree & 9 & 8.26 & 8 & 9.52 & 17 & 8.81 & 0.2450 & 0.8850 \\
\hline Somewhat agree & 11 & 10.09 & 7 & 8.33 & 18 & 9.33 & & \\
\hline Agree & 89 & 81.65 & 69 & 82.14 & 158 & 81.87 & & \\
\hline
\end{tabular}

Facility and environment
Q1 I found the operative laboratory and the operative clinic free of distractions and conducive to study

\begin{tabular}{|l|c|c|c|c|c|c|c|c|}
\hline Disagree & 1 & 0.92 & 0 & 0.00 & 1 & 0.52 & 0.8040 & 0.6690 \\
\hline Somewhat agree & 3 & 2.75 & 2 & 2.38 & 5 & 2.59 & & \\
\hline Agree & 105 & 96.33 & 82 & 97.62 & 187 & 96.89 & & \\
\hline Total & & & & & & & &
\end{tabular}




\begin{tabular}{|l|l|l|l|l|l|l|l|l|l|} 
& 109 & 100.0 & 84 & 100.0 & 193 & 100.0 & & \\
\hline
\end{tabular}

Likewise, no statistical difference in responses were seen between the levels ( $p>0.05)$, except for Q2 in the Learning domain (see Table 2).

Table 2. Comparison of item responses on the scale based on level 


\begin{tabular}{|c|c|c|c|c|c|c|c|c|c|}
\hline Variables & Items & $\begin{array}{c}10^{\text {th }} \\
\text { level }\end{array}$ & $\%$ & $\begin{array}{l}11^{\text {th }} \\
\text { level }\end{array}$ & $\%$ & $\begin{array}{l}12^{\text {th }} \\
\text { level }\end{array}$ & $\%$ & $\mathrm{c}^{2}$ & $\begin{array}{c}\mathrm{p}- \\
\text { value }\end{array}$ \\
\hline \multirow[t]{12}{*}{ Learning objectives } & \multicolumn{9}{|c|}{ Q1 I have understood the learning objectives of operative course. } \\
\hline & Disagree & 0 & 0.00 & 0 & 0.00 & 0 & 0.00 & 4.0150 & 0.1340 \\
\hline & $\begin{array}{l}\text { Somewhat } \\
\text { agree }\end{array}$ & 15 & 22.73 & 14 & 22.58 & 7 & 10.77 & & \\
\hline & Agree & 51 & 77.27 & 48 & 77.42 & 58 & 89.23 & & \\
\hline & \multicolumn{9}{|c|}{ Q2 I have gained knowledge and skills consistent with the learning objectives. } \\
\hline & Disagree & 9 & 13.64 & 1 & 1.61 & 1 & 1.54 & 13.6640 & $.008^{*}$ \\
\hline & $\begin{array}{l}\text { Somewhat } \\
\text { agree }\end{array}$ & 11 & 16.67 & 13 & 20.97 & 8 & 12.31 & & \\
\hline & Agree & 46 & 69.70 & 48 & 77.42 & 56 & 86.15 & & \\
\hline & \multicolumn{9}{|c|}{ Q3 The course has clarified my role as a student. } \\
\hline & Disagree & 0 & 0.00 & 0 & 0.00 & 0 & 0.00 & 2.9670 & 0.2270 \\
\hline & $\begin{array}{l}\text { Somewhat } \\
\text { agree }\end{array}$ & 7 & 10.61 & 4 & 6.45 & 2 & 3.08 & & \\
\hline & Agree & 59 & 89.39 & 58 & 93.55 & 63 & 96.92 & & \\
\hline \multirow[t]{12}{*}{ Course materials } & \multicolumn{9}{|c|}{ Q1 The course materials (slides, lectures, assignments, quiz, etc.) are easy to follow. } \\
\hline & Disagree & 5 & 7.58 & 2 & 3.23 & 1 & 1.54 & 6.9370 & 0.1390 \\
\hline & $\begin{array}{l}\text { Somewhat } \\
\text { agree }\end{array}$ & 12 & 18.18 & 8 & 12.90 & 5 & 7.69 & & \\
\hline & Agree & 49 & 74.24 & 52 & 83.87 & 59 & 90.77 & & \\
\hline & \multicolumn{9}{|c|}{ Q2 The complexity and level of detail of the materials are appropriate. } \\
\hline & Disagree & 0 & 0.00 & 2 & 3.23 & 0 & 0.00 & 4.9420 & 0.2930 \\
\hline & $\begin{array}{l}\text { Somewhat } \\
\text { agree }\end{array}$ & 4 & 6.06 & 3 & 4.84 & 2 & 3.08 & & \\
\hline & Agree & 62 & 93.94 & 57 & 91.94 & 63 & 96.92 & & \\
\hline & \multicolumn{9}{|c|}{$\begin{array}{l}\text { Q3 The course materials, including resources, are essential to my success in operative } \\
\text { dentistry }\end{array}$} \\
\hline & Disagree & 5 & 7.58 & 2 & 3.23 & 2 & 3.08 & 7.0210 & 0.1350 \\
\hline & Disagree & 8 & 12.12 & 2 & 3.23 & 3 & 4.62 & & \\
\hline & $\begin{array}{l}\text { Somewhat } \\
\text { agree }\end{array}$ & 53 & 80.30 & 58 & 93.55 & 60 & 92.31 & & \\
\hline \multirow[t]{9}{*}{ Content relevance } & \multicolumn{9}{|c|}{ Q1 I shall be able to apply what I learned during this course in the future as a dentist. } \\
\hline & Disagree & 0 & 0.00 & 0 & 0.00 & 0 & 0.00 & 1.0290 & 0.5980 \\
\hline & $\begin{array}{l}\text { Somewhat } \\
\text { agree }\end{array}$ & 1 & 1.52 & 1 & 1.61 & 0 & 0.00 & & \\
\hline & Agree & 65 & 98.48 & 61 & 98.39 & 65 & 100.0 & & \\
\hline & \multicolumn{9}{|c|}{ Q2 I have obtained the necessary knowledge and skills to become a successful dentist. } \\
\hline & Disagree & 1 & 1.52 & 1 & 1.61 & 0 & 0.00 & 1.0380 & 0.9040 \\
\hline & $\begin{array}{l}\text { Somewhat } \\
\text { agree }\end{array}$ & 3 & 4.55 & 3 & 4.84 & 3 & 4.62 & & \\
\hline & Agree & 62 & 93.94 & 58 & 93.55 & 62 & 95.38 & & \\
\hline & \multicolumn{9}{|c|}{$\begin{array}{l}\text { Q3 I know where to find answers to the questions that may arise in my role as a } \\
\text { dentist. }\end{array}$} \\
\hline
\end{tabular}




\section{Instructor}

knowledge

Instructor delivery and style

\begin{tabular}{|l|c|c|c|c|c|c|c|c|} 
Disagree & 4 & 6.06 & 4 & 6.45 & 2 & 3.08 & 3.3830 & 0.4960 \\
\hline $\begin{array}{l}\text { Somewhat } \\
\text { agree }\end{array}$ & 7 & 10.61 & 3 & 4.84 & 3 & 4.62 & & \\
\hline Agree & 55 & 83.33 & 55 & 88.71 & 60 & 92.31 & & \\
\hline
\end{tabular}

Q1 My learning was enriched by the instructor's knowledge.

\begin{tabular}{|l|c|c|c|c|c|c|c|c|}
\hline Disagree & 2 & 3.03 & 3 & 4.84 & 1 & 1.54 & 1.3970 & 0.8450 \\
\hline $\begin{array}{l}\text { Somewhat } \\
\text { agree }\end{array}$ & 3 & 4.55 & 2 & 3.23 & 2 & 3.08 & & \\
\hline Agree & 61 & 92.42 & 57 & 91.94 & 62 & 95.38 & & \\
\hline
\end{tabular}

Q2 My learning was enriched by the experience of the instructor and the examples shared in the class.

\begin{tabular}{|l|c|c|c|c|c|c|c|c|}
\hline Disagree & 4 & 6.06 & 2 & 3.23 & 1 & 1.54 & 2.2680 & 0.6870 \\
\hline $\begin{array}{l}\text { Somewhat } \\
\text { agree }\end{array}$ & 5 & 7.58 & 4 & 6.45 & 6 & 9.23 & & \\
\hline Agree & 57 & 86.36 & 56 & 90.32 & 58 & 89.23 & & \\
\hline
\end{tabular}

Q1 I was well engaged during the operative course.

\begin{tabular}{|l|c|c|c|c|c|c|c|c|}
\hline Disagree & 5 & 7.58 & 1 & 1.61 & 2 & 3.08 & 5.3830 & 0.2500 \\
\hline $\begin{array}{l}\text { Somewhat } \\
\text { agree }\end{array}$ & 6 & 9.09 & 4 & 6.45 & 2 & 3.08 & & \\
\hline Agree & 55 & 83.33 & 57 & 91.94 & 61 & 93.85 & & \\
\hline
\end{tabular}

Q2 I found it easy to be actively involved in the learning process of the operative course.

\begin{tabular}{|l|c|c|c|c|c|c|c|c|}
\hline Disagree & 5 & 7.58 & 1 & 1.61 & 2 & 3.08 & 5.8610 & 0.2100 \\
\hline $\begin{array}{l}\text { Somewhat } \\
\text { agree }\end{array}$ & 8 & 12.12 & 5 & 8.06 & 3 & 4.62 & & \\
\hline Agree & 53 & 80.30 & 56 & 90.32 & 60 & 92.31 & & \\
\hline
\end{tabular}

Q3 I had ample opportunity to ask questions and receive answers during my course.

\begin{tabular}{|l|c|c|c|c|c|c|c|c|}
\hline Disagree & 3 & 4.55 & 1 & 1.61 & 0 & 0.00 & 4.0700 & 0.3970 \\
\hline $\begin{array}{l}\text { Somewhat } \\
\text { agree }\end{array}$ & 4 & 6.06 & 2 & 3.23 & 3 & 4.62 & & \\
\hline Agree & 59 & 89.39 & 59 & 95.16 & 62 & 95.38 & & \\
\hline
\end{tabular}

Q4 I had ample opportunity to practice and demonstrate skills that I had learnt.

\begin{tabular}{|l|c|c|c|c|c|c|c|c|}
\hline Disagree & 7 & 10.61 & 3 & 4.84 & 5 & 7.69 & 3.1090 & 0.5400 \\
\hline $\begin{array}{l}\text { Somewhat } \\
\text { agree }\end{array}$ & 6 & 9.09 & 3 & 4.84 & 3 & 4.62 & & \\
\hline Agree & 53 & 80.30 & 56 & 90.32 & 57 & 87.69 & & \\
\hline
\end{tabular}

Q5 I was comfortable with the pace of the operative sessions in the course.

\begin{tabular}{|l|c|c|c|c|c|c|c|c|}
\hline Disagree & 9 & 13.64 & 3 & 4.84 & 4 & 6.15 & 6.7850 & 0.1480 \\
\hline $\begin{array}{l}\text { Somewhat } \\
\text { agree }\end{array}$ & 8 & 12.12 & 5 & 8.06 & 3 & 4.62 & & \\
\hline Agree & 49 & 74.24 & 54 & 87.10 & 58 & 89.23 & & \\
\hline
\end{tabular}

Q6 I was comfortable with the length of the operative sessions in the course.

\begin{tabular}{|l|c|c|c|c|c|c|c|c|}
\hline Disagree & 10 & 15.15 & 3 & 4.84 & 4 & 6.15 & 6.5150 & 0.1640 \\
\hline $\begin{array}{l}\text { Somewhat } \\
\text { agree }\end{array}$ & 8 & 12.12 & 5 & 8.06 & 5 & 7.69 & &
\end{tabular}




\begin{tabular}{|c|c|c|c|c|c|c|c|c|c|}
\hline & Agree & 48 & 72.73 & 54 & 87.10 & 56 & 86.15 & & \\
\hline \multirow[t]{5}{*}{$\begin{array}{l}\text { Facility and } \\
\text { environment }\end{array}$} & \multicolumn{9}{|c|}{$\begin{array}{l}\text { Q1 I found the operative laboratory and the operative clinics free of distractions and } \\
\text { conducive to study. }\end{array}$} \\
\hline & Disagree & 1 & 1.52 & 0 & 0.00 & 0 & 0.00 & 3.4950 & 0.4790 \\
\hline & $\begin{array}{l}\text { Somewhat } \\
\text { agree }\end{array}$ & 3 & 4.55 & 1 & 1.61 & 1 & 1.54 & & \\
\hline & Agree & 62 & 93.94 & 61 & 98.39 & 64 & 98.46 & & \\
\hline & Total & 66 & 100.0 & 62 & 100.0 & 65 & 100.0 & & \\
\hline
\end{tabular}

$* \mathrm{p}<0.05$

It was observed that the percentage of respondents with disagreement was higher to Q2 in the Learning domain as compared to the other domains among the three levels. The results of the average total score for all questionnaire domains and comparison between genders showed that mean scores for each domain are closer to the maximum scores, respectively. Furthermore, there was no statistically significant difference in the average satisfaction scores between genders ( $p>0.05)$. (see Table 3 ).

Table 3. Comparison of total satisfaction and its dimensions based on gender by Mann-Whitney U test

\begin{tabular}{|c|c|c|c|c|c|c|}
\hline Components & Summary & Male & Female & Total & Z-value & $\mathrm{P}$-value \\
\hline \multirow{2}{*}{$\begin{array}{l}\text { Learning objectives } \\
(\text { Total score possible }=9 \text { ) }\end{array}$} & Mean & 8.45 & 8.49 & 8.47 & \multirow[t]{2}{*}{-0.5718} & \multirow[t]{2}{*}{0.5674} \\
\hline & SD & 1.03 & 1.08 & 1.05 & & \\
\hline \multirow{2}{*}{$\begin{array}{l}\text { Course materials } \\
\text { (Total score possible }=9 \text { ) }\end{array}$} & Mean & 8.51 & 8.62 & 8.56 & \multirow[t]{2}{*}{-0.2599} & \multirow[t]{2}{*}{0.7949} \\
\hline & SD & 1.16 & 1.02 & 1.10 & & \\
\hline \multirow{2}{*}{$\begin{array}{l}\text { Content relevance } \\
\text { (Total score possible }=9 \text { ) }\end{array}$} & Mean & 8.70 & 8.82 & 8.75 & \multirow[t]{2}{*}{-0.3938} & \multirow[t]{2}{*}{0.6937} \\
\hline & SD & 0.92 & 0.58 & 0.79 & & \\
\hline \multirow{2}{*}{$\begin{array}{l}\text { Instructor knowledge } \\
\text { (Total score possible }=6 \text { ) }\end{array}$} & Mean & 5.73 & 5.77 & 5.75 & \multirow[t]{2}{*}{-0.0286} & \multirow[t]{2}{*}{0.9772} \\
\hline & SD & 0.83 & 0.73 & 0.79 & & \\
\hline \multirow{2}{*}{$\begin{array}{l}\text { Instructor delivery and style } \\
(\text { Total score possible }=18 \text { ) }\end{array}$} & Mean & 16.94 & 16.76 & 16.87 & \multirow[t]{2}{*}{-0.1650} & \multirow[t]{2}{*}{0.8689} \\
\hline & SD & 2.52 & 2.65 & 2.57 & & \\
\hline \multirow{2}{*}{$\begin{array}{l}\text { Facility and environment } \\
(\text { Total score possible }=3 \text { ) }\end{array}$} & Mean & 2.95 & 2.98 & 2.96 & \multirow[t]{2}{*}{-0.1560} & \multirow[t]{2}{*}{0.8761} \\
\hline & SD & 0.25 & 0.15 & 0.21 & & \\
\hline \multirow{2}{*}{$\begin{array}{l}\text { Total satisfaction } \\
\text { (Total score possible }=54 \text { ) }\end{array}$} & Mean & 51.29 & 51.44 & 51.36 & \multirow[t]{2}{*}{-0.3028} & \multirow[t]{2}{*}{0.7620} \\
\hline & SD & 5.93 & 5.14 & 5.59 & & \\
\hline
\end{tabular}


The overall results for total satisfaction and its dimensions show there that there is no statistically significant difference in average satisfaction scores among student levels ( $p>0.05)$. However, in the Learning domain, level 10 respondents had significantly lower total satisfaction average scores as compared to scores of respondents in other levels $(p<0.05)$. (see Table 4$)$.

Table 4. Comparison of total satisfaction and its dimensions by level using Kruskal-Wallis test

\begin{tabular}{|c|c|c|c|c|c|c|}
\hline Components & Summary & $10^{\text {th }}$ level & $11^{\text {th }}$ level & $12^{\text {th }}$ level & $\mathrm{H}$-value & P-value \\
\hline \multirow{2}{*}{$\begin{array}{l}\text { Learning objectives } \\
\text { (Total score possible }=9 \text { ) }\end{array}$} & Mean & 8.23 & 8.47 & 8.71 & \multirow[t]{2}{*}{4.3690} & \multirow[t]{2}{*}{$0.0370^{*}$} \\
\hline & SD & 1.31 & 0.94 & 0.79 & & \\
\hline \multirow{2}{*}{$\begin{array}{l}\text { Course materials } \\
(\text { Total score possible }=9 \text { ) }\end{array}$} & Mean & 8.33 & 8.60 & 8.75 & \multirow[t]{2}{*}{0.8260} & \multirow[t]{2}{*}{0.3630} \\
\hline & SD & 1.29 & 1.08 & 0.85 & & \\
\hline \multirow{2}{*}{$\begin{array}{l}\text { Content relevance } \\
\text { (Total score possible }=9 \text { ) }\end{array}$} & Mean & 8.68 & 8.73 & 8.85 & \multirow[t]{2}{*}{0.1900} & \multirow[t]{2}{*}{0.6630} \\
\hline & $\mathrm{SD}$ & 0.86 & 0.91 & 0.57 & & \\
\hline \multirow{2}{*}{$\begin{array}{l}\text { Instructor knowledge } \\
\text { (Total score possible }=6 \text { ) }\end{array}$} & Mean & 5.70 & 5.74 & 5.82 & \multirow[t]{2}{*}{0.0260} & \multirow[t]{2}{*}{0.8730} \\
\hline & SD & 0.89 & 0.83 & 0.63 & & \\
\hline \multirow{2}{*}{$\begin{array}{l}\text { Instructor delivery and style } \\
\text { (Total score possible }=18 \text { ) }\end{array}$} & Mean & 16.21 & 17.23 & 17.18 & \multirow[t]{2}{*}{0.0670} & \multirow[t]{2}{*}{0.7960} \\
\hline & SD & 3.30 & 1.97 & 2.10 & & \\
\hline \multirow{2}{*}{$\begin{array}{l}\text { Facility and environment } \\
(\text { Total score possible }=3 \text { ) }\end{array}$} & Mean & 2.92 & 2.98 & 2.98 & \multirow[t]{2}{*}{0.0010} & \multirow[t]{2}{*}{0.9730} \\
\hline & SD & 0.32 & 0.13 & 0.12 & & \\
\hline \multirow{2}{*}{$\begin{array}{l}\text { Total satisfaction } \\
\text { (Total score possible }=54 \text { ) }\end{array}$} & Mean & 50.08 & 51.74 & 52.29 & \multirow[t]{2}{*}{3.3140} & \multirow[t]{2}{*}{0.0690} \\
\hline & SD & 7.11 & 4.62 & 4.39 & & \\
\hline
\end{tabular}

${ }^{*} \mathrm{p}<0.05$

The association between levels of satisfaction (High and Low) based on demographic profile showed statistically significant difference among the three levels $(10,11$, and 12$)$ but no difference was seen in terms of the gender of the students. ( $p>0.05)$ (see Table 5$)$.

Table 5. Association between levels of satisfaction based on demographic profile 


\begin{tabular}{|c|c|c|c|c|c|c|c|}
\hline \multirow[t]{2}{*}{ Profile } & \multicolumn{5}{|c|}{ Levels of satisfaction } & \multirow[t]{2}{*}{$\mathrm{c} 2$} & \multirow[t]{2}{*}{$\mathrm{p}$-value } \\
\hline & Low level & $\%$ & High level & $\%$ & Total & & \\
\hline \multicolumn{8}{|l|}{ Level } \\
\hline Level 10 & 21 & 31.82 & 45 & 68.18 & 66 & 6.1993 & $0.0450 *$ \\
\hline Level 11 & 13 & 20.97 & 49 & 79.03 & 62 & & \\
\hline Level 12 & 9 & 13.85 & 56 & 86.15 & 65 & & \\
\hline \multicolumn{8}{|l|}{ Gender } \\
\hline Male & 23 & 21.10 & 86 & 78.90 & 109 & 0.2010 & 0.6539 \\
\hline Female & 20 & 23.81 & 64 & 76.19 & 84 & & \\
\hline Total & 43 & 22.28 & 150 & 77.72 & 193 & & \\
\hline
\end{tabular}

$* \mathrm{p}<0.05$

\section{Discussion}

Students at three different levels of the operative dentistry course in Saudi Arabia were participants of a survey on their professional satisfaction regarding the teaching and curriculum of the course. These students had already undergone preclinical training and were into clinical training. Clinical-based education is a multi-factorial process wherein the students implement the theoretical knowledge they gain in preclinical training on patients. Dentistry is an important field of medical science, and hence, enhancing the quality of dental clinical education directly improves the oral/dental health of people. Dentistry is a clinical major in which adequate skills and training are highly important in graduate students' performance, which would consequently promote the oral and dental health systems. Because the educational system, equipment, and performance of tutors are different in dental schools, the results of this study cannot be generalized to other dental schools. Therefore, surveying students' opinion about the quality of clinical education in different dentistry colleges can surely improve the educational programs and clinical training quality.

In the Kingdom of Saudi Arabia, most colleges follow the semester-type curriculum. Each year has two semesters, each comprising 14 weeks of actual teaching and 4 weeks of practical/clinical and final theory exams. Operative dentistry starts from level 4 of the dental course. Level 4 to level 6 are primarily preclinical courses where, the students are introduced to the materials they will be using and work in simulated laboratories learning different cavity design preparations and restorations with different restorative materials. In addition to these, they have E-Learning assignments on the recent advances in material sciences and techniques. The course is regularly updated in keeping with the recent trends to introduce students to the latest technology and familiarize them with the same. Levels $8,10,11$, and 12 involve students implementing their skills on patients under the direct supervision of their supervisors. As mentioned earlier, it is very important to evaluate and assess whether the teaching methods are successful. 
Each course has its own learning objectives, which are specified and described at the beginning in the course specifications. During the first lecture, the learning objectives are explained to the students and the same is evaluated at the end of the semester by testing whether students have obtained the requisite knowledge and skills consistent with the learning objectives and are clear about what is expected of them. In the current study, it was observed that most of the respondents agreed that the Learning objectives were met during the course (Tables 1 and 2).

During the course, the students are exposed to a variety of teaching materials and strategies such as lectures, power point presentations, and assessment criteria such as quizzes, online assignments, and continuous evaluation of their preclinical work/clinical work. These need to be evaluated and assessed to understand whether they are consistent with the learning objectives to achieve the intended goal. The results of this study indicated that all the three items under the Course Material domain showed that neither the level nor the gender led to any statistical difference in responses ( $p>0.05)$ (Tables 1 and 2$)$.

After training, it is critical to understand the implications of how the student applies his training as a dentist in society. The aim of the learning process is to produce an independent thinking dentist who will be able to apply the necessary knowledge and skills using his or her rationale to be a successful dentist. The relevance of the course is reflected when the intended goals are achieved. The results of this study have indicated that most respondents agreed that the course training increased their level of confidence to work as an independent dentist. The results for all three items under Course Relevance domain showed no statistical difference in responses regardless of gender or level $(p>0.05)$. A majority of the respondents $(>88 \%)$ agreed that the course was relevant as seen from the training (Tables 1 and 2 ).

Instructors play an important role in the shaping the attitude and enthusiasm of the students. The knowledge and skills of the instructor along with his or her experience is a vital component of the teaching process, especially in clinical sciences. Students often get influenced and motivated by the instructor. The results of this study showed most of the respondents $(>80 \%)$ had a positive opinion about the role of instructors. There was no statistical difference seen in terms of gender or level $(p>0.05)$ (Tables 1 and 2).

Since operative dentistry is a clinical subject, the importance of the clinical facilities and the environment needs to be emphasized. The clinical setup, the availability of the latest materials, and the instruments and their utilization during the clinical training period plays an important role in the education of the students. Most respondents in this study agreed $(>90 \%)$ that the facility and conduciveness of the operative dentistry clinics were satisfactory. There was no statistical difference based on gender or level ( $p>0.05$ ) (Tables 1 and 2). However, it is interesting to note that at a higher level, satisfaction was greater.

Analyzing the association of between the levels of satisfaction from the perspective of gender and level, it was interesting to observe there was a statistical difference in terms of the level of the students (Table 5). Level 10 students recorded a lower level of satisfaction (31.82\%) whereas levels 11 and 12 recorded a high level of satisfaction (68.18\%). There was no statistical difference in the gender group $(p>0.05)$ (Table 5). 


\section{Conclusions}

Feedback and satisfaction of students toward the teaching they are offered in the operative dentistry course and the curriculum would be an important tool in dental education. The study findings show that most students of Levels 10,11 , and 12 were satisfied with the teaching and the curriculum of operative dentistry in Saudi Arabia. The level of satisfaction was also observed to be higher at the higher levels of the course. With rapid innovation in terms of materials and techniques in the field of operative dentistry, the curriculum needs to be updated and continuously assessed by students to understand their satisfaction as they are the final recipients of the educational process.

\section{Declarations}

Ethics approval and consent to participate: Ethical clearance for this study was obtained from the Institutional Review Board of King Khalid University College of Dentistry, Approval No IRB/KKUCOD/ETH/2019-20/012 dated 25/12/2019. Informed written consent was obtained from all the participants of the questionnaire study.

Consent for publication: Not applicable.

Competing interests: The author declares that there are no competing interests.

Availability of data and materials: The datasets during and/or analysed during the current study available from the corresponding author on reasonable request.

Funding: Nil.

Author Contributions: MAA has contributed to the conception of the work, has drafted the work and in preparing and writing the manuscript. MAA has read and approved the manuscript.

Acknowledgements: The author would like to thank Dr S B Javali for the statistical analysis and Dr Shashit Shetty Bavabeedu for the help in acquisition of the data. The author would also like to thank all the dental students who participated in the study.

Author Information: Mohammed Al Qarni has completed his Board Certification and Specialty Certificate in Restorative Dentistry from SCHS, Riyadh, Saudi Arabia in 2012. He completed his Master of Clinical Restorative and Esthetic Dentistry from Pacific University, CA, USA \& accordingly gained his Diplomate of MFDRCSI, Dublin, Ireland in 2014.

He is former Vice-Dean and is currently working as Associate Professor in the department of Restorative Dental Sciences at College of Dentistry, King Khalid University, Abha, Kingdom of Saudi Arabia.

\section{References}


1. Kirkpatrick D. Techniques for Evaluating Training Programs. Revisiting Kirkpatrick's Four-Level Model. Training and Development, v50 n1 p54-59 Jan 1996.

2. Kirkpatrick DL, Kirkpatrick JD. Implementing the Four Levels: A Practical Guide for Effective Evaluation of Training Programs. San Francisco, CA: Berrett-Koehler Publishers; 2007.

3. El Ansari W, Moseley L. You get what you measure: Assessing and reporting student satisfaction with their health and social care educational experience. Nurse Educ Today 2011;31(2):173-178.

4. Wiers-Jenssen J, Stensaker B, Grogaard JB. Student satisfaction: Towards an empirical deconstruction of the concept. Qual Higher Educ 2002;8:183-195.

5. Brown G, Manogue M, Rohlin M. Assessing attitudes in dental education: Is it worthwhile? Br Dent J 2002;193(12):703-707.

6. Divaris K, Barlow PJ, Chendea SA, Cheong WS, Dounis A, Dragan IF, et al. The academic environment: the students' perspective. Eur J Dent Educ 2008;12 Suppl 1:120-130.

7. Shepard LA. The role of assessment in a learning culture. Educ Res 2000;29(7):4-14.

8. Pariseau SE, McDaniel JR. Assessing service quality in schools of business. Int J Qual Reliabil Manag 1997;14(3):204-218.

9. Henzi D, Davis E, Jasinevicius R, Hendricson W. In the students' own words: What are the strengths and weaknesses of the dental school curriculum? J Dent Educ 2007;71(5):632-645.

10. Greenfield, V., Shelton, S., Balkovich, E., Davis, J., Adamson, D. Adult learning principles and training evaluation. Appendix F. In: The Federal Voting Assistance Program and the Road Ahead: Achieving Institutional Change through Analysis and Collaboration Santa Monica, CA: RAND Corporation; 2015:171-186.

\section{Supplementary Files}

This is a list of supplementary files associated with this preprint. Click to download.

- QuestionnaireSurveyProfessionalSatisfaction.pdf 